\title{
Dynamics of a coefficient of friction during non-stationary sliding of a parabolic indenter on visco-elastic foundation
}

Cite as: AIP Conference Proceedings 1783, 020041 (2016); https://doi.org/10.1063/1.4966334

Published Online: 10 November 2016

Andrey V. Dimaki, and Valentin L. Popov

\section{ARTICLES YOU MAY BE INTERESTED IN}

Fractal characteristics of seismic process in rock mass surrounding the excavation at mining.

Mathematical modelling and analysis

AIP Conference Proceedings 1783, 020048 (2016); https://doi.org/10.1063/1.4966341

Sliding simulation of automotive brake primary contact with variable amounts of copper and graphite nanoparticles

AIP Conference Proceedings 1783, 020044 (2016); https://doi.org/10.1063/1.4966337

Theoretical study of strength of elastic-plastic water-saturated interface under constrained shear

AIP Conference Proceedings 1783, 020042 (2016); https://doi.org/10.1063/1.4966335

\section{Lock-in Amplifiers up to $600 \mathrm{MHz}$

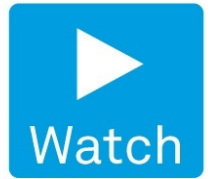

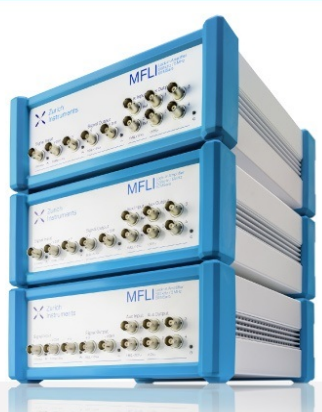




\title{
Dynamics of a Coefficient of Friction during Non-Stationary Sliding of a Parabolic Indenter on Visco-Elastic Foundation
}

\author{
Andrey V. Dimaki ${ }^{1, a)}$ and Valentin L. Popov² \\ ${ }^{1}$ Institute of Strength Physics and Materials Science SB RAS, Tomsk, 634055 Russia \\ ${ }^{2}$ Berlin University of Technology, Berlin, 10623 Germany \\ a) Corresponding author: dav@ispms.tsc.ru
}

\begin{abstract}
We have studied the coefficient of friction between a rigid parabolic indenter and a visco-elastic Kelvin foundation under step-wise change of sliding velocity. We have obtained analytical estimations for normal and tangential forces in a contact and their limiting values during transition process that occurs after a jump of sliding velocity. The results of numerical simulation are in good agreement with analytical estimates.
\end{abstract}

Since Coulomb [1] it has been known that non-stationary regime of sliding may result in a non-linear time dependence of forces acting to contacting bodies. In his famous works Dieterich reported a time-dependent static friction law [2] and later formulated his law of non-stationary sliding for geological materials [3], introducing an internal "parameter of state". Basing on these results, Ruina has analyzed the area of applicability of singleparametrical rate-and-state law, and suggested a number of differential equations that can describe experimental data $[4,5]$. At present time, rate-and-state laws are being used in wide range of studies, including the analysis of earthquake generation and seismic cycle [6], simulation of friction of lubricated surfaces [7], fracture propagation [8] etc.

In the works mentioned above the contacting bodies are considered as elastic or elastic-plastic. At that, a detailed study of the role of viscosity of a material and, more widely, a role of time-dependent mechanical response of contacting bodies in non-stationary sliding still remains of interest, despite a number of experimental works in this field (see, for example [9, 9]. In the present paper, we consider a non-stationary tangential contact between a viscoelastic body and rigid parabolic indenter at constant indentation depth that is a governing parameter [11]. We use the "single-asperity" approach, assuming that contact patches are situated far enough from each other and do not interact. The latter allows to essentially simplify the problem and to obtain analytical expressions for contact forces, shape of the contact area and, thus, for a coefficient of friction.

We use the following assumptions: (a) the elastomer represents a simple incompressible Kelvin body, which is characterized by its static shear modulus and viscosity, (b) the surface of the elastomer is assumed to be initially plane and friction-less, (c) we consider a contact between single parabolic asperity, that is absolutely rigid, and Kelvin foundation, (d) no adhesion or capillarity effects are taken into account, (e) we consider a one-dimensional model. Despite these simplifications, the developed model allows us to provide a number of analytical estimations and observe non-trivial behavior in non-stationary regime of sliding.

We use the method of dimensionality reduction (MDR) for simulation $[12,13]$. Following this method, the elastomer was modeled as a row of independent elements with a small spacing $\Delta x$, each element consisting of a spring with normal stiffness $\Delta k=4 G \Delta x$ and a dashpot having the damping constant $\Delta d=4 \eta \Delta x$, where $G$ is the shear modulus and $\eta$ is the viscosity of the elastomer [13].

Recently, Popov and Hess [13] have obtained a complete analytic solution of the contact problem between a conical indenter and visco-elastic foundation in stationary regime of sliding. Basing on their approach, we start our analysis from obtaining the estimates for a contact area and contact forces and then continue with the consideration of a transitional process. 


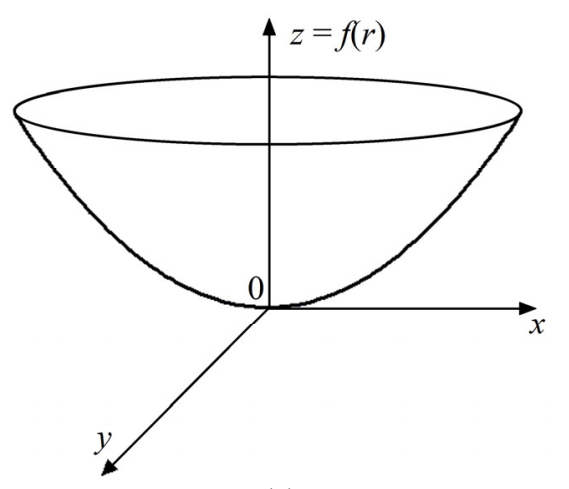

(a)

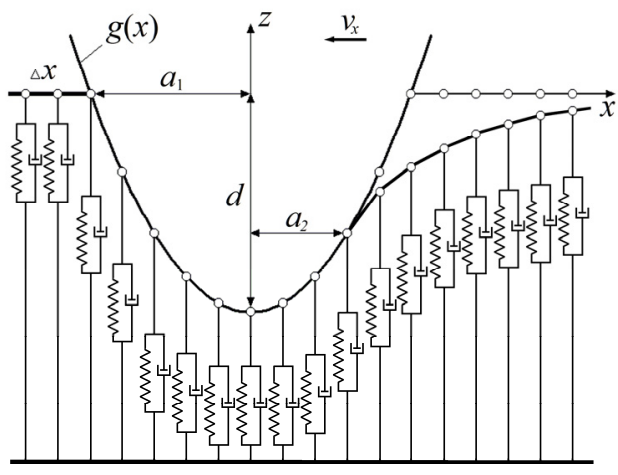

(b)

FIGURE 1. (a) Initial 3-dimensional profile of a parabolic indenter, (b) schematic of the contact between one-dimensional sliding indenter and Kelvin's foundation

Let us consider a rigid parabolic indenter $\tilde{z}=f(r)=r^{2} / 2 R$, where $\tilde{z}$ is the coordinate normal to the contact plane, and $r$ is the in-plane polar radius (Fig. 1a).

The one-dimensional MDR-image of this profile, according to the rules of the MDR is

$$
\tilde{z}=g(x)=x^{2} / R .
$$

Following the methodology of the work [13], we suggest the profile being pressed into a visco-elastic foundation to a constant depth of $d$ and moved tangentially with the velocity $v$ so that its form is described at time $t$ by the equation

$$
\tilde{z}=g(x+v t)=g(\tilde{x}) .
$$

As stated above, we assume that the elastomer is a simple visco-elastic material (Kelvin's body), which can be modeled as parallel-connected springs and dashpots (Fig. 1b). If the three-dimensional medium is characterized by the shear modulus $G$ and the viscosity $\eta$, then the stiffness $\Delta k_{z}$ and the damping coefficient $\Delta \gamma$ of an element of the visco-elastic foundation must be chosen as follows [12]:

$$
\Delta k_{z}=4 G \Delta x, \Delta \gamma=4 \eta \Delta x
$$

where $\Delta x$ is the spatial size of an element of the foundation. In order to simplify the analysis, it is useful to introduce the coordinate $\tilde{x}$ in the frame of reference that moves with the rigid indenter. Thus, the coordinates of the boundary of the contact area are $\tilde{x}=-a_{1}$ and $\tilde{x}=a_{2}$ (Fig. 1a). Vertical displacements $u_{z}$ in the entire contact area are determined by the purely geometric condition

$$
u_{z}(x, t)=d-g(x+v t)=d-g(\tilde{x}) .
$$

The vertical velocities are

$$
\frac{\partial u_{z}(x, t)}{\partial t}=-\frac{\partial g(x+v t)}{\partial t}=-v g^{\prime}(\tilde{x})
$$

and the normal force acting on a single element is

$$
f_{N}(\tilde{x})=\Delta k_{z} u_{z}+\Delta \gamma \dot{u}_{z}=4\left[G(d-g(\tilde{x}))-\eta v g^{\prime}(\tilde{x})\right] \Delta x .
$$

The left boundary of the contact area is determined by the condition $u_{z}\left(-a_{1}\right)=0$ and the right boundary by the condition of zero normal force $f_{N}\left(a_{2}\right)=0$. Under the conditions of stationary sliding with constant velocity $v$ the coordinates of the contact are can be determined as follows [13]:

$$
a_{1}=\sqrt{R d}, a_{2}=\sqrt{v^{2} \tau^{2}+R d}-v \tau,
$$

where we introduce the relaxation time

$$
\tau=\eta / G
$$


Changes of sliding velocity lead to variation of the distance of detachment $a_{2}=a_{2}(t)$. In that case the total normal force is a function of time:

$$
\begin{aligned}
F_{N}(t) & =4 \int_{-a_{1}}^{a_{2}(t)}\left[G(d-g(\tilde{x}))-\eta v g^{\prime}(\tilde{x})\right] \mathrm{d} \tilde{x}=4 G \int_{-a_{1}}^{a_{2}(t)}\left(d-\tilde{x}^{2} / R-2 \tau v \tilde{x} / R\right) \mathrm{d} \tilde{x} \\
= & 4 G\left[d\left(a_{1}+a_{2}(t)\right)-\frac{1}{3 R}\left(a_{1}^{3}-a_{2}^{3}(t)\right)+\frac{\tau \nu}{R}\left(a_{1}^{2}-a_{2}^{2}(t)\right)\right] .
\end{aligned}
$$

The time dependence of the total tangential force can be written as follows:

$$
\begin{aligned}
F_{x}(t) & =4 \int_{-a_{1}}^{a_{2}(t)} g^{\prime}(\tilde{x})\left[G(d-g(\tilde{x}))-\eta v g^{\prime}(\tilde{x})\right] \mathrm{d} \tilde{x}=8 G \int_{-a_{1}}^{a_{2}(t)}\left[\frac{\tilde{x} d}{R}-\frac{\tilde{x}^{3}}{R^{2}}-\frac{2 \tau v \tilde{x}^{2}}{R^{2}}\right] \mathrm{d} \tilde{x} \\
& =\frac{8 G}{R^{2}}\left[\frac{R d}{2}\left(a_{1}^{2}-a_{2}^{2}(t)\right)-\frac{1}{2}\left(a_{1}^{4}-a_{2}^{4}(t)\right)+\frac{2 \tau v}{3}\left(a_{1}^{3}+a_{2}^{3}(t)\right)\right] .
\end{aligned}
$$

For a stationary sliding with constant velocity $v$ the distance of detachment $a_{2}(t)=$ const is determined by the Eq. (7). Obviously, a time dependence of the coefficient of friction is completely determined by the relation of tangential and normal forces,

$$
\mu(t)=F_{x}(t) / F_{N}(t)
$$

which, in turn, are determined with a distance of detachment $a_{2}(t)$. As one can see from the Eq. (7), the distance of detachment tends to zero with increase of sliding velocity but never achieves it. Nevertheless, it is possible to obtain estimations of normal and tangential forces assuming that $a_{2} \rightarrow 0$ at $v \rightarrow \infty$. From that point of view,

$$
\begin{aligned}
& F_{N}^{\mathrm{inf}} \rightarrow 4 G\left[\frac{2}{3} R^{1 / 2} d^{3 / 2}+\tau v d\right], \\
& F_{x}^{\mathrm{inf}} \rightarrow 4 G\left[\frac{d^{2}}{2}+\frac{4}{3} \tau v \sqrt{d^{3} / R}\right] .
\end{aligned}
$$

The value of coefficient of friction $\mu^{\text {inf }}$ that is achieved at $v \rightarrow \infty$ is:

$$
\mu^{\inf }=F_{x}^{\inf } / F_{N}^{\text {inf }} \approx \frac{4}{3} \sqrt{d / R}
$$

It is also useful to estimate the values of force jumps at the step-wise change of the sliding velocity. Let us suggest that in the moment of a velocity change $t=t_{0}$ the distance of detachment still remains the same as before $a_{2}\left(t=t_{0}\right)=\sqrt{\tau^{2} v^{2}+R d}-\tau v$, but the sliding proceeds with a new velocity $v_{1}$. After simple transformations of the Eq. (9) we have a maximal value of normal force that occurs immediately after the velocity change:

$$
F_{N}^{\text {jump }}=F_{N}\left(t_{0}, v=v_{1}\right)=4 G\left[d\left(a_{1}+a_{2}\left(t_{0}\right)\right)-\frac{1}{3 R}\left(a_{1}^{3}-a_{2}^{3}\left(t_{0}\right)\right)+\frac{\tau v_{1}}{R}\left(a_{1}^{2}-a_{2}^{2}\left(t_{0}\right)\right)\right] \text {. }
$$

Applying the same approach to the tangential force (see the Eq. (10)) we have:

$$
F_{x}^{\text {jump }}=F_{x}\left(t_{0}, v=v_{1}\right)=\frac{8 G}{R^{2}}\left[\frac{R d}{2}\left(a_{1}^{2}-a_{2}^{2}\left(t_{0}\right)\right)-\frac{1}{2}\left(a_{1}^{4}-a_{2}^{4}\left(t_{0}\right)\right)+\frac{2 \tau v_{1}}{3}\left(a_{1}^{3}+a_{2}^{3}\left(t_{0}\right)\right)\right] .
$$

In order to demonstrate the behavior of the developed model we have calculated the contact forces, the coefficient of friction and the distance of detachment $a_{2}(t)$ numerically. We have considered the "start-stop" test, when the initial value of the velocity $v$ is high enough $\left(a_{2} \rightarrow 0\right)$ and the value of the velocity after the step-wise change is close to zero, $v_{1}=v \times 10^{-4}\left(a_{2} \rightarrow a_{1}\right)$. Such test allows obtaining the limiting values of the forces so that any values of initial and final sliding velocities will lead to the forces between mentioned limiting values. In the Figs. $2 \mathrm{a}$ and $2 \mathrm{~b}$ the time dependencies of normal and tangential forces, acting on the indenter, are shown. It is seen that a step-wise change of the sliding velocity leads to an immediate jump of a force, followed by a "relaxation" to a new stable value. It is interesting to note that the coefficient of friction doesn't have the same jump (see Fig. 2c). While the coefficient of friction changes from its maximal value determined by the Eq. (14), the distance of detachment $a_{2}(t)$ goes from a value near zero to the $a_{2}(v \rightarrow 0) \approx \sqrt{R d}=a_{1}$ (Fig. 2d). 


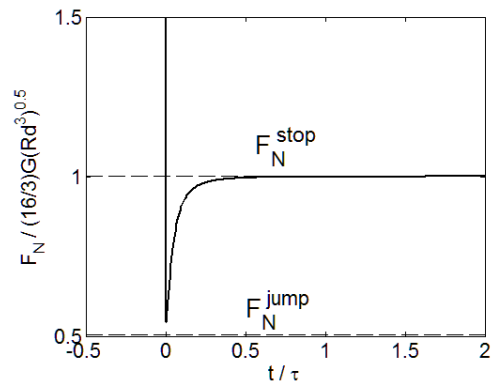

(a)

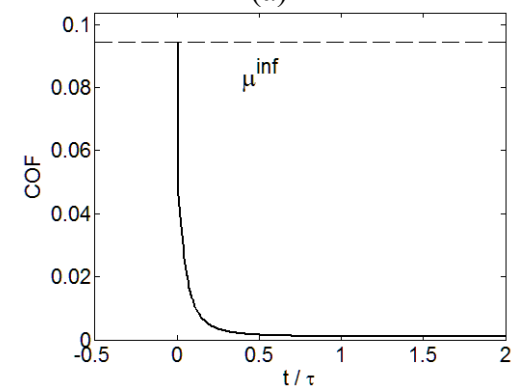

(c)

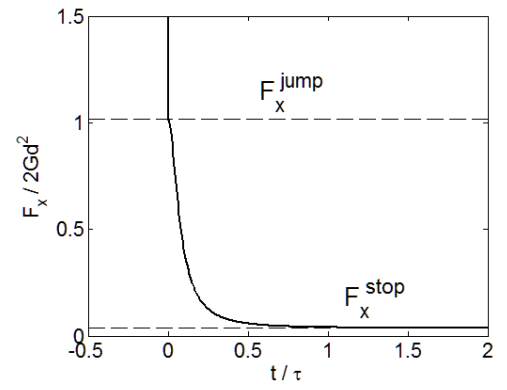

(b)

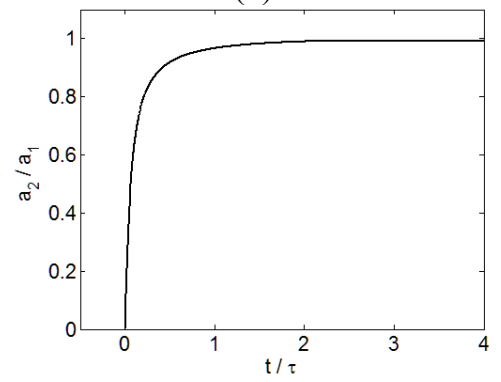

(d)

FIGURE 2. Evolution of a contact under step-wise change of the sliding velocity: (a) the normal force, (b) the tangential force, (c) the coefficient of friction, (d) the distance of detachment

The results of numerical simulation show that the forces in contact and the coefficient of friction demonstrate a complicated, non-linear behavior under a variation of the sliding velocity even in simplest conditions: a parabolic indenter sliding on a linear Kelvin's foundation. We have obtained the analytical estimates for the values characterizing the evolution of the coefficient of friction: stationary and peak values of reaction forces and the size of contact area. Comparison of the analytical estimates with the result of direct numerical simulation of the contact problem has shown very good quantitative agreement between them.

\section{ACKNOWLEDGMENTS}

The authors thank the Russian Science Foundation (Project 14-19-00718) for financial support.

\section{REFERENCES}

1. C. A. Coulomb, Theorie des machines simple (Bachelier, Paris, 1821).

2. J. H. Dieterich, Trans. Am. Geophys. Union 51, 423 (1970).

3. J. H. Dieterich, J. Geophys. Res. 84, 2161-2168 (1979).

4. A. Ruina, J. Geophys. Res. 88, 10359-10370 (1983).

5. J. R. Rice and A. L. Ruina, J. Appl. Mech. 50, 343-349 (1983).

6. C. Marone, Nature 391, 69-72 (1997).

7. J. M. Carlson and A. A. Batista, Phys. Rev. E 53, 4153-4165 (1996).

8. A. Bizarri and M. Cocco, J. Geophys. Res. 117, B02314 (2012).

9. J. H. Dieterich and B. D. Kilgore, Pure Appl. Geophys. 143, 283-302 (1994).

10. H. Zeng, Polymer Anhesion, Friction and Lubrication (John Wiley \& Sons, Hoboken, 2013).

11. V. L. Popov, Phys. Mesomech. 19(2), 115-122 (2016).

12. V. L. Popov and M. Hess, Facta Universit. Mech. Engng. 12, 1-14 (2014).

13. V. L. Popov and M. Heß, Method of Dimensionality Reduction in Contact Mechanics and Friction (Springer, Berlin, 2014). 